\title{
Regiospecific synthesis of 6-halouridine derivatives: an effective method for coupling sterically hindered pyrimidine bases to ribose
}

\author{
Daniel J. Blackburn*, Greggory T. Kent, and Weiming Wu* \\ Department of Chemistry and Biochemistry, San Francisco State University, San Francisco, CA 94132 USA
}

\section{ARTICLE INFO}

Article history:

Received

Received in revised form

Accepted

Available online

Keywords:

6-halouracil

nucleoside synthesis

ribosylation

pyrimidine silylation

sterically hindered pyrimidine

\section{ABSTRACT}

6-Halouridine derivatives were synthesized regospecifically through the coupling of N3protected 6-halouracil to a ribose derivative. The combination of the silylating reagent $\mathrm{N}, \mathrm{O}$ bis(trimethylsilyl)acetamide and Lewis acid catalyst trimethylsilyl trifluoromethanesulfonate is unique in their ability to facilitate the coupling of sterically hindered pyrimidine bases to ribose to form nucleoside derivatives.

2009 Elsevier Ltd. All rights reserved.

\section{Introduction}

In the course of our investigation of the mechanism of orotidine 5'-monophosphate decarboxylase (ODCase), we have decided to study the interaction of different functional groups at the 6-position of uridine 5'-monophosphate (UMP). Prior investigations yielded interesting insights on the reaction mechanism and some of the derivatives were reported to have antimalarial or anticancer activities. ${ }^{1-5}$ We are especially interested in the 6-haloUMP derivatives due to the covalent modification of ODCase by 6-iodoUMP. ${ }^{2}$ It would be interesting to investigate how the size of different halogens at $\mathrm{C}-6$ affects the reaction of these UMP analogues with ODCase.

6-IodoUMP was synthesized through the lithiation of the 6position of protected uridine followed by iodination with elemental iodine (Scheme 1). ${ }^{2}$ Unfortunately, effort to chlorinate and brominate the 6-carbanion intermediate did not produce the desired halogenated product.

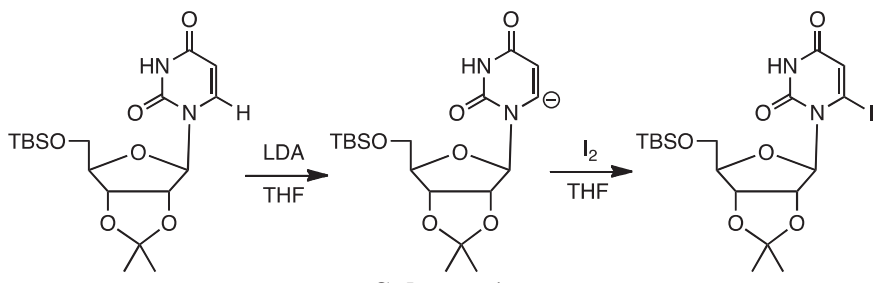

Scheme 1

Nucleotide analogues are usually prepared from the phosphorylation of corresponding nucleosides, which are synthesized from the coupling of purine or pyrimidine with ribose derivatives. However, in the reaction of 6-chlorouracil with ribose derivatives, it was discovered that ribosylation at N-3 was favored and that the yield for the desired N-1 ribosylation product was very poor. We therefore planned to protect N-3 with benzyl group so that the coupling reaction would only occur at $\mathrm{N}-1$. In this Letter, we describe the efficient synthesis of 6chloro- and 6-bromouridine derivatives through the coupling of these protected 6-halouracils to ribose derivatives.

\section{Results and Discussion}

The synthesis started with the readily available $\mathrm{N}$ benzylbarbituric acid (1) as shown in Scheme 2. NBenzylbarbituric acid has been synthesized from malonic acid under acidic conditions or from malonic ester under basic conditions. ${ }^{6,7}$ The base-catalyzed reaction was found to work much better in our laboratory. The reaction of oxyphosphorus chloride or bromide occurred regiospecifically at the carbonyl group furthest away from the benzyl group. ${ }^{8-10}$ The relative position of the chlorine and benzyl groups in $\mathbf{2 a}$ was confirmed by ${ }^{1} \mathrm{H}-{ }^{15} \mathrm{~N}$ HSQC and ${ }^{1} \mathrm{H}-{ }^{13} \mathrm{C}$ HMBC NMR spectroscopy.

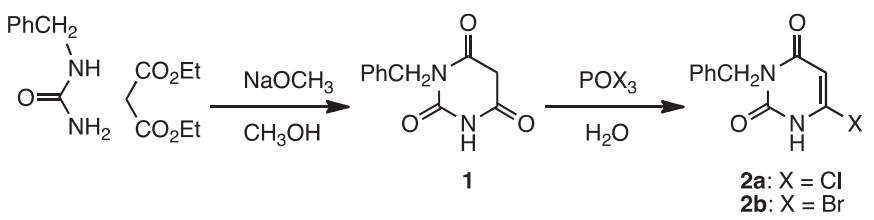

Scheme 2

\footnotetext{
* Corresponding author. Tel.: +0-415-338-1436; fax: +0-415-338-2384; e-mail: djblack987@gmail.com; wuw@sfsu.edu.
} 
It should be noted that the reaction carried out with pure oxyphosphorus halides only worked on a very small scale and the addition of $N, N$-dimethylaniline did not improve the reaction. ${ }^{11,12}$ However, addition of a small amount of water allowed the reaction to proceed on gram-scale in reasonable yields $(71 \%$ and $73 \%$ for chlorination and bromination, respectively. ${ }^{7,10}$

In the second step, 3-benzyl-6-halouracil (2) was coupled to a ribose derivative to produce the nucleoside derivatives. The coupling of pyrimidine and purine bases to ribose derivatives has been investigated extensively and numerous methods have been developed. ${ }^{13}$ However, the coupling step involving 6-substituted uracils 2 turned out to be problematic. The Vorbrüggen modification of the Hilbert-Johnson method has been the most widely used coupling reaction to attach the ribose or other sugar units to purine or pyrimidine bases in the preparation of nucleoside derivatives. ${ }^{14-16}$ In this reaction, the pyrimidine or purine bases are usually silylated with hexamethyldisilazane and the silylated bases then react with a tetraacylated ribose in the presence of a Lewis acid catalyst such as $\mathrm{SnCl}_{4}, \mathrm{C}_{4} \mathrm{H}_{9} \mathrm{SO}_{3} \mathrm{~K}$, and TMSOTf (trimethylsilyl trifluoromethanesulfonate). ${ }^{14-16}$ However, the reactions were found to make very little progress towards completion. Two protocols that were reported to successfully promote the synthesis of 6-methyluridine nucleoside derivatives, which also contains a sterically hindered reaction site, failed as well. ${ }^{17,18}$ The reason for lack of reactivity seen with 6-halouracils 2 may be of both steric and electronic nature.

We then turned to coupling methods involving nucleophilic attack on 1-bromoribosyl benzoate by the sodium or mercury salts of the halouracil anion. ${ }^{19-23}$ Unfortunately, none of the reactions we attempted gave any indication of nucleoside formation. The direct fusion of 1,2,3,5-O-tetraacetylribofuranose with uracils 2 also failed to yield any product. ${ }^{24}$

We decided to further experiment with the Vorbrüggen method with different silylating agents. ${ }^{25,26}$ Gratifyingly, when the combination of $\mathrm{N}, \mathrm{O}$-bis(trimethylsilyl)acetamide as a silylating agent and TMSOTf as the Lewis acid catalyst was employed, the coupling of halouracil 2 with the widely used ribose derivative $\mathbf{3}$ (1-O-acetyl-2,3,5-O-tribenzoyl)- $\beta$-Dribofuranose) was quite successful (Scheme 3). ${ }^{26}$ The yields for nucleoside derivatives $\mathbf{4 a}$ and $\mathbf{4 b}$ were $84 \%$ and $82 \%$, respectively. ${ }^{27}$ The structure of the products was confirmed by ${ }^{1} \mathrm{H}$ and ${ }^{13} \mathrm{C}$ NMR spectroscopy and the positions of substitution on uracil were confirmed by ${ }^{1} \mathrm{H}-{ }^{15} \mathrm{~N}$ HMBC NMR spectroscopy. The nucleoside derivatives 4 can be readily deprotected and converted to corresponding nucleotides as reported. ${ }^{2,28,29}$ Their reaction with ODCase will be investigated in due course.

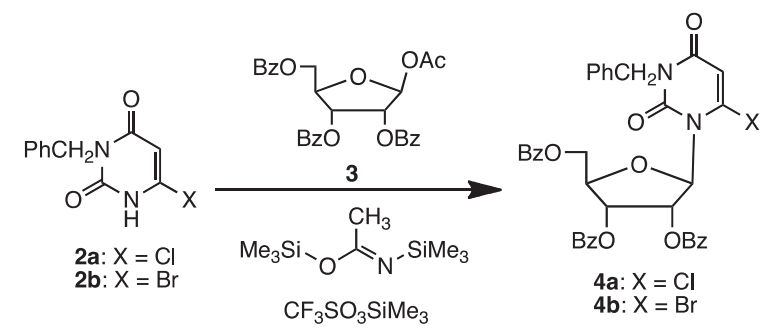

Scheme 3

\section{Conclusion}

In summary, 3-benzyl-6-halouracils were successfully prepared from $N$-benzylbarbituric acid in good yields. The coupling of these 6-halouracil bases to ribose was found to be problematic but an efficient strategy combining $\mathrm{N}, \mathrm{O}-$ bis(trimethylsilyl)acetamide as a silylating agent and TMSOTf as a Lewis acid catalyst was successfully employed. The coupling method described here should be of interest in the synthesis of nucleoside derivatives involving sterically hindered pyrimidine or purine bases.

\section{Acknowledgments}

This investigation was supported by the National Institutes of Health (Grant No. SC1 GM095419) (W.W.) and CSUPERB Presidents' Commission Scholarship (D.J.B.). We thank Dr. Mark Swanson for obtaining the 2D NMR spectra. The NMR facility was funded by the National Science Foundation (DUE9451624 and DBI 0521342). We also thank Professors Ihsan Erden and Taro Amagata at SFSU for helpful discussions and David Gutierrez and Eric Suon for technical assistance.

\section{References and Notes}

1. Fujihashi, M.; Bello, A. M.; Poduch, E.; Wei, L.; Annedi, S. C.; Pai, E. F.; Kotra, L. P. J. Am. Chem. Soc. 2005, 127, 15048-15050.

2. Bello, A. M.; Poduch, E.; Fujihashi, M.; Amani, M.; Li, Y.; Crandall, I.; Hui, R.; Lee, P.; Kain, K. C.; Pai, E. F.; Kotra, L. P. J. Med. Chem. 2007, 50, 915-921.

3. Bello, A. M.; Poduch, E.; Liu, Y.; Wei, L.; Crandall, I.; Wang, X. Dyanand, C.; Kain, K. C.; Pai, E. F.; Kotra, L. P. J. Med. Chem. 2008, 51, 439-448.

4. Bello, A. M.; Konforte, D.; Poduch, E.; Furlonger, C.; Wei, L.; Liu, Y.; Lewis, M.; Pai, E. F.; Paige, C. J.; Kotra, L. P. J. Med. Chem. 2009, 52, 1648-1658.

5. Poduch, E.; Bello, A. M.; Tang, S.; Fujihashi, M.; Pai, E. F.; Kotra, L. P. J. Med. Chem. 2006, 49, 4937-4945.

6. Bruckmann, G.; Isaacs, S. D. J. Am. Chem. Soc. 1949, 71, 390-392.

7. Jurok, R.; Cibulka, R.; Dvorakova, H.; Hampl, F.; Hodacova, J. Eur. J. Org. Chem. 2010, 5217-5224.

8. Nübel, G.; Pfleiderer, W. Chem. Ber. 1962, 95, 1605-1614.

9. $\quad$ Pflerderer, W.; Deiss, H. Israel J. Chem. 1968, 6, 603-614.

10. The halogenation reactions were found to be regiospecific in our laboratory, though non-regioselective results were also reported: Nagamatsu, T.; Yamasaki, H.; Yoneda, F. Heterocycles 1994, 37, 1147-1164

11. Bench, A.; Russell, Jr., P.; Fox, J. J. J. Am. Chem. Soc. 1954, 76, 6073-6077.

12. Arnott, E. A.; Chan, L. C.; Cox, B. G.; Meyrick, B.; Phillips, A. J. Org. Chem. 2011, 76, 1653-1661.

13. For a review, see: Vorbrüggen, H.; Ruh-Pohlenz, C. Handbook of Nucleoside Synthesis; Wiley: New York, 2001.

14. Niedballa, U.; Vorbrüggen, H. Angew. Chem. Int. Ed. 1970, 9, 461462.

15. Vorbrüggen, H.; Bennua, B. Chem. Ber. 1981, 114, 1279-1286.

16. Challenger, S.; Dessi, Y.; Fox, D. E.; Hesmondhalgh, L. C.; Pascal, P.; Pettman, A. J.; Smith, J. D. Org. Process Res. Dev. 2008, 12, 575-583.

17. Felczak, K.; Drabikowska, A. K.; Vilpo, J. A.; Kulikowski, T.; Shugar, D. J. Med. Chem. 1996, 39, 1720-1728.

18. Beigelman, L. et al. International Patent WO 96/18736, 1996.

19. Nagamatsu, T.; Ma, J.; Akiba, S. Nucleic Acids Symp. Series 2008, 52, 561-562. doi: 10.1093/nass/nrn284.

20. Kazimierczuk, Z.; Cottam, H. B.; Revankar, G. R.; Robins, R. K. J. Am. Chem. Soc. 1984, 106, 6379-6382.

21. Marasco, Jr., C. J.; Pera, P. J.; Spiess, A. J.; Bernacki, R.; Sufrin, J. R. Molecules 2005, 10, 1015-1020.

22. Davoll, J.; Lowy, B. A. J. Am. Chem. Soc. 1951, 73, 1650-1655.

23. Burrows, W. J.; Armstrong, D. J.; Skoog, F.; Hecht, S. M.; Boyle, J. T. A.; Leonard, N. J.; Occolowitz, J. Biochemistry 1969, 8, 30713076.

24. Witkowski, J. T.; Robins, R. K.; Sidwell, R. W.; Simon, L. N. J. Med. Chem. 1972, 15, 1150-1154. 
25. Bambury, R. E.; Feeley, D. T.; Lawton, G. C.; Weaver, J. M.; Wemple, J. J. Med. Chem. 1984, 27, 1613-1621.

26. Wunderlich, C. H.; Spitzer, R.; Santner, T.; Fauster, K.; Tollinger, M.; Kreutz, C. J. Am. Chem. Soc. 2012, 134, 7558-7569.

27. Experimental details: All reagents were obtained from commercial sources and used without further purification. Typical experimental procedures are described below using the syntheses of 3-benzyl-6bromouracil (2b) and 1-(2,3,5-tri-O-benzoyl- $\beta$-D-ribofuranosyl)-3benzyl-6-chlorouracil (4b) as examples. NMR spectra were obtained in DMSO- $\mathrm{d}_{6}$ on a Bruker $500 \mathrm{MHz}$ instrument.

3-Benzyl-6-bromouracil (2b): Water $(0.2 \mathrm{~g}, 11.5 \mathrm{mmol})$ was added dropwise to a stirred mixture of N-benzylbarbituric acid (2.00 g, 9.7 $\mathrm{mmol})$ in phosphorus oxybromide $(13.2 \mathrm{~g}, 90.2 \mathrm{mmol})$ in a roundbottom flask at $0{ }^{\circ} \mathrm{C}$. After the mixture was stirred for 1.5 hours at $100{ }^{\circ} \mathrm{C}$, the round-bottom flask was chilled in an ice bath. Ice water was added slowly with constant stirring. Once all solid had disappeared, the solution was neutralized with aqueous potassium hydroxide. The precipitated solid was filtered and recrystallized from acetonitrile to give $\mathbf{2 b}$ as an off-white solid $(1.90 \mathrm{~g}, 73 \%)$.

1-(2,3,5-tri-O-benzoyl- $\beta$-D-ribofuranosyl)-3-benzyl-6-bromouracil (4b): Bromouracil $2 \mathbf{b}(114 \mathrm{mg}, 0.4 \mathrm{mmol})$ and 1-O-acetyl-2,3,5-Otribenzoyl)- $\beta$-D-ribofuranose $(3,204 \mathrm{mg}, 0.4 \mathrm{mmol})$ was mixed in a suspension in anhydrous acetonitrile $(3.5 \mathrm{~mL})$ in a flame-dried twonecked flask. Bis(trimethylsilyl)acetamide (122 mg, $0.6 \mathrm{mmol})$ was added dropwise and the mixture was stirred at room temperature for $30 \mathrm{~min}$. The reaction was cooled to $0{ }^{\circ} \mathrm{C}$ and trimethylsily trifluoromethanesulfonate $(133 \mathrm{mg}, 0.6 \mathrm{mmol})$ was added. Upon warming up to room temperature, the reaction was stirred at $60{ }^{\circ} \mathrm{C}$ for 2 hours. The reaction mixture turned golden yellow and TLC showed the reaction was complete. The solvent was evaporated and the oily residue was dissolved in methylene chloride, and the.organic phase was washed with saturated sodium bicarbonate solution, dried over sodium sulfate, and evaporated to give a redish oil. The product was further purified by column chromatography with methylene chloride as an eluent to give $\mathbf{4 b}$ as a clear thick oil ( $248 \mathrm{mg}, 82 \%)$.

28. Zimmerman, M. N.; Nemeroff, N. H.; Bock, C. W.; Bhat, K. L. Heterocycles 2000, 53, 205-211.

29. Levine, H. L.; Brody, R. S.; Westheimer, F. H. Biochemistry 1980, 19, 4993-4999. 


\section{Graphical Abstract}

Regiospecific synthesis of 6-halouridine derivatives:

Leave this area blank for abstract info. an effective method for coupling sterically hindered pyrimidine bases to ribose

Daniel J. Blackburn*, Greggory T. Kent, and Weiming Wu*

Department of Chemistry and Biochemistry, San Francisco State University, San Francisco, CA 94132 USA

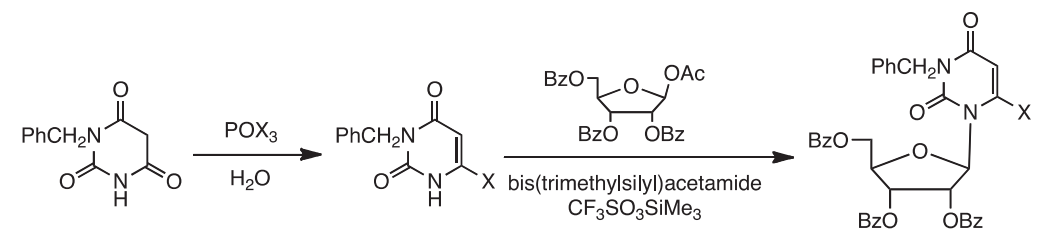

